\title{
Isokinetic assessment of knee flexors and extensors in professional soccer players
}

\author{
J.L. Croisier ${ }^{\mathrm{a}}$, V. Reveillon ${ }^{\mathrm{a}}$, J.M. Ferret ${ }^{\mathrm{b}}$, T. Cotte ${ }^{\mathrm{b}}$, M. Genty ${ }^{\mathrm{c}}$, N. Popovich ${ }^{\mathrm{d}}$, Mohty Filho ${ }^{\mathrm{e}}$, \\ J.E. Faryniuk ${ }^{\mathrm{e}}$, S. Ganteaume ${ }^{\mathrm{a}}$ and J.M. Crielaard ${ }^{\mathrm{a}}$ \\ ${ }^{\mathrm{a}}$ Department of Physical Medicine and Rehabilitation, CHU Sart Tilman, Liege, Belgium \\ ${ }^{\mathrm{b}}$ Center of Sports Medicine, Lyon-Gerland, France \\ ${ }^{\mathrm{c}}$ Center La Roseraie, St Adresse, France \\ ${ }^{\mathrm{d}}$ Standard de Liege football Club, Liege, Belgium \\ ${ }^{\mathrm{e}}$ Tuiuti of Parana University, Curitiba, Brazil
}

\begin{abstract}
Muscle injuries are among the most common insults in soccer players and result from multifactorial origins. In recent studies [1,2], we focused on hamstring muscle strains because of the high frequency of re-injury and persistent complaints after return to athletic activities. This study attempted to define the thigh muscle profile in professional soccers and to identify preseason isokinetic strength variables as predictors of hamstring muscle strain.
\end{abstract}

Seventy-seven professional soccer players (age: 26 \pm 4 years, weight: $76 \pm 5 \mathrm{~kg}$, height: $180 \pm 5 \mathrm{~cm}$ ) recruited from 4 elite teams (in Belgium, Brazil and France) were included in the study. All the participating Centers used a Cybex Norm dynamometer and a standardized preseason isokinetic protocol consisting in concentric exertions (angular speeds of $60 \mathrm{deg} / \mathrm{sec}$ and $240 \mathrm{deg} / \mathrm{sec}$ ) of both knee flexor and quadriceps muscles; afterward, flexors were subjected to eccentric angular speeds of $30 \mathrm{deg} / \mathrm{sec}$ and $120 \mathrm{deg} / \mathrm{sec}$. The nature of muscle abnormalities was determined using statistically selected cutoffs: bilateral differences of $15 \%$ or more, concentric ratio less than 0.47 , and mixed ratio (eccentric flexors / concentric quadriceps) less than 0.80 . Thereafter, players were observed for 9 months and we recorded the severity and frequency of new hamstring muscle injuries throughout the subsequent competitive season.

Focusing on the quadriceps in the concentric mode at $60 \mathrm{deg} / \mathrm{sec}$, the whole group of players averaged 3.03
$( \pm 0.42) \mathrm{N} . \mathrm{m} / \mathrm{kg}$, with a $7 \%$ difference between extreme team values. Flexors in eccentric at $30 \mathrm{deg} / \mathrm{sec}$ reached on average $2.32( \pm 0.64) \mathrm{N} . \mathrm{m} / \mathrm{kg}$, with a $34 \%$ difference between extreme team results. Referring to the selected limits, $55 \%$ of the whole group of players were isokinetically imbalanced and result analysis confirmed the discriminating character of the eccentric trial and the specificity of the original mixed ratio [2]. During the longitudinal follow-up, seven players sustained clinically diagnosed hamstring muscle injuries that caused them to miss playing time (more than 4 weeks). Among them, six presented isokinetic preseason abnormalities. These results allow us to fix the risk factor of hamstring injury at:

- $15 \%$ in the presence of strength imbalance (6 injuries in 41 imbalanced players),

- only 3\% in the absence of strength abnormality (1 injury in 36 normal players).

Soccer players were isokinetically characterized by a great interindividual variability of flexor and extensor muscle performances. Assuming the multifactorial origin of muscular injuries, we emphasize the importance of strength imbalances as a major risk factor. Soccers with imbalances appeared 5 times more likely to sustain a hamstring strain than those without the imbalance. Consequently, preseason isokinetic assessment may allow a preventive approach of that problem. 


\section{References}

[1] J.L. Croisier and J.M. Crielaard, Hamstring muscle tear with recurrent complaints: an isokinetic profile, Isokinetics Exerc Sci 8 (2000), 175-180.
[2] J.L. Croisier et al., Hamstring muscle strain recurrence and strength performance disorders, Am J Sports Med 30 (2002), 199-203. 